\title{
Dietary fat intake associated with bone mineral density among visfatin genotype in obese people
}

\author{
Leila Khorrami-Nezhad ${ }^{1}$, Khadijeh Mirzaei ${ }^{2 *}$, Zhila Maghbooli $^{1}$ and Seyed Ali Keshavarz ${ }^{3}$ \\ ${ }^{1}$ Osteoporosis Research Center, Endocrinology and Metabolism Clinical Sciences Institute, University of Medical Sciences, PO \\ Box 14114-13137, Tehran, Iran \\ ${ }^{2}$ Department of Community Nutrition, School of Nutritional Sciences and Dietetics, Tehran University of Medical Sciences \\ (TUMS), PO Box 14155-6117, Tebran, Iran \\ ${ }^{3}$ Department of Clinical Nutrition, School of Nutritional Sciences and Dietetics, Tehran University of Medical Sciences (TUMS), \\ PO Box 14155-6117, Tehran, Iran
}

(Submitted 17 February 2017 - Final revision received 26 August 2017 - Accepted 27 September 2017)

\begin{abstract}
Osteoporosis and adipose tissue are closely related with many contradictions. Visfatin is an adipokine that is related to osteoporosis and adiposity. This nutrigenomics study examined the interaction between visfatin genotypes and dietary fat intake, with regard to bone mineral density (BMD) among an obese population. In this cross-sectional study, 336 subjects were enrolled; the mean age was 38.25 (sD 11.69) years and the mean BMI was 31.79 (sD 4.77$) \mathrm{kg} / \mathrm{m}^{2}$. Laboratory measurements were lipid profile, insulin and fasting blood sugar. Bone density measurements were assessed by dual-energy X-ray absorptiometry. Dietary data were collected through a 3-d 24-h dietary recall. Genotyping for visfatin gene SNP (rs2110385) was performed by the PCR-restriction fragment length polymorphism method. The frequency of GG, GT and TT genotypes were 33.9248 .51 and $17.54 \%$, respectively, and $86.6 \%$ of participants were women. The results showed that subjects with TT genotypes had significantly higher lumbar BMD, $T$ score and $z$ score $(P<0.0001)$. After categorisation by percentage of fat intake $(30 \%$ of total energy content as a cut-off point), no interaction was found, but when categorised by fat types, we found an interaction between visfatin genotypes and dietary PUFA intake in terms of the hip $T$ score and $z$ score $(P=0.043, B=-0.08 ; P=0.04, B=-0.078$, respectively). There was a significant relationship between high PUFA intake and lower energy and protein intake. When participants were categorised by median PUFA intake (22.8g), it was concluded that subjects with GG genotype who had high PUFA-intake diets had lower hip $z$ scores and $T$ scores, unlike the other genotypes.
\end{abstract}

\section{Key words: Gene-diet interaction: Visfatin genotypes: Bone density: Dietary fat intakes}

As the population ages, osteoporosis and obesity are increasingly becoming major public health concerns. Both are considered as progressive diseases of complex aetiology. The first is characterised by low bone mineral density (BMD) and increased risk of frequent fractures, whereas the second is defined as the excessive accumulation and storage of fat. Previous studies have indicated that these disorders were interrelated ${ }^{(1-3)}$. Many studies have reported that adipose tissue influences osteoporosis and osteoporotic fractures; therefore, adipose tissue maybe considered as a link between obesity and $\mathrm{BMD}^{(3-7)}$. Several investigations have found significant positive ${ }^{(8,9)}$ or negative ${ }^{(6)}$ effects of obesity on bone status at different regions. Adipose tissue, through the secretion of compounds, has an influence on bone density.

Recent studies have suggested that visfatin has a major function on the relationship between adipose tissue and osteoporosis ${ }^{(10)}$. Visfatin, an adipocyte-secreted peptide, which is highly expressed in visceral and subcutaneous tissue ${ }^{(11)}$, was found to have a controversial effect on bone density ${ }^{(12,13)}$, and also on bone metabolism. The insulin-mimetic effect of Visfatin also has a major role in osteoblast function ${ }^{(21)}$. On the other hand, the visfatin genotype is correlated to lipid serum concentration and bone density $^{(14,15)}$.

It seems that visfatin has a regulatory role in lipid metabo$\operatorname{lism}^{(16)}$, as well being correlated with dietary fat intake ${ }^{(17-19)}$, which could suggest a link between the visfatin genotype and BMD. In order to clarify the effects of individual differences, visfatin genotypes were considered. This nutrigenomics study examined the interaction between visfatin genotypes and dietary fat intake, with regard to BMD among the obese population.

\section{Methods}

\section{Study population}

This cross-sectional study included 336 individuals with a mean age of 38.25 (sD 11.69) years and mean BMI of 31.79 (sD 4.77$) \mathrm{kg} / \mathrm{m}^{2}$,

Abbreviation: BMD, bone mineral density.

* Corresponding author: K. Mirzaei, fax +98 21 88984861, email mirzaei_kh@tums.ac.ir 
all of whom were referred to the endocrine clinic of Shariati hospital to routinely assess their health status associated with overweight and obesity from July to December 2015. None of the subjects had diabetes, heart disease, acute or inflammatory disease, a medical history of hypertension, hormone therapy or alcohol and drug abuse. All patients received adequate information about the study and written consent forms were obtained before enrolment. The protocol was approved by the ethics committee of Tehran University of Medical Sciences. The local ethics committee of Tehran University of Medical Sciences approved the study (ID: 94-01-161-28473).

\section{Body composition measurement}

Complete body composition analysis was done, whereby for all cases a Body Composition Analyzer BC-418MA (Tanita) was used. Therefore, in principle, subjects were barefoot when they were assessed by this device. To prevent a possible discrepancy in measured values, we avoided taking measurements after vigorous exercise and waited until the subject was sufficiently rested. As changes in body-water distribution and body temperature can have a major impact on measurements, they were performed in the morning in a fasting condition (always urinating before taking measurements, etc.) to get a more accurate result of the measurements every single time. For each participant, accomplished anthropometric parameters including weight (to the nearest $0.1 \mathrm{~kg}$ ) and height (to the nearest $0.1 \mathrm{~cm}$ ) were measured while the subjects were fasting and measured in light clothing without shoes. BMI was calculated for all patients as body weight (in $\mathrm{kg}$ ) divided by height (in $\mathrm{m}$ ) squared.

\section{Bone mineral density measurements}

All the subjects underwent BMD measurements by dual-energy $\mathrm{X}$-ray absorptiometry of the lumbar spine (vertebrae L2-L4) and total hip. The results are presented as absolute values in $\mathrm{g} / \mathrm{cm}^{2}$ and as standard deviation units ( $T$ score) based on comparison with the reference BMD (30-year-old young woman).

\section{Genotyping}

Visfatin genotypes were evaluated with the official symbol NAMPT and described in NCBI with gene ID (10135), which is located in Chromosome 7 (7q22.2). In this study, a variation on the visfatin gene promoter region $(-4689 \mathrm{G} / \mathrm{T})$, which is registered in NCBI as SNP rs2110385, was detected by a PCR-based Restriction Fragment Length Polymorphism (RFLP) assay. Flexi Gen DNA extraction kit (QIAGEN) was used for DNA extraction from human blood. All procedures were described in the study by Körner $e t a l .{ }^{(20)}$. Genomic DNA from all subjects was analysed for the presence of the $G$ or $T$ nucleotide at $-4689 \mathrm{G} / \mathrm{T}$ of the visfatin gene by a PCR-based RFLP assay. A PCR amplification of a 951-bp fragment of the upstream region of the visfatin gene containing the polymorphism was carried out using left primer, 5' CACTTCTTTATTTTGGGGTTGC 3', and right primer, 5' GCAGTCTGGGAGCTCTGG 3', designed and used before PC. A $25-\mu \mathrm{l}$ PCR reaction mixture containing $2 \mu \mathrm{l}$ of genomic DNA, $5 \mu \mathrm{l}$ of deoxynucleotide triphosphates, $5 \mu \mathrm{l}$ of forward and reverse primers, $5 \mu \mathrm{l}$ of $10 \times$ buffer, $1.5 \mu \mathrm{l}$ of $\mathrm{MgCl}_{2}$ and $0.5 \mu \mathrm{l}$ of Taq polymerase was mixed and placed in a thermal cycler. After denaturing for $10 \mathrm{~min}$ at $95^{\circ} \mathrm{C}$, the DNA was amplified for thirty-five cycles at $95^{\circ} \mathrm{C}$ for $60 \mathrm{~s}, 58^{\circ} \mathrm{C}$ for $60 \mathrm{~s}$ and $72^{\circ} \mathrm{C}$ for $60 \mathrm{~s}$, followed by a 7 -min extension at $72^{\circ} \mathrm{C}$. A positive control containing genomic DNA and a negative control containing everything except DNA were included in the PCR experiment. A quantity of $5 \mu \mathrm{l}$ of each PCR product, including the controls, were verified on a $2 \%$ agarose gel to ensure that the expected 951-bp product was generated. A restriction digest for the DNA fragment was carried out using the AluI restriction enzyme. A quantity of $15 \mu \mathrm{l}$ of the PCR product was digested for $16 \mathrm{~h}$ overnight at $37^{\circ} \mathrm{C}$ with 1 unit of AluI (mi-E0101L; Metabion). The RFLP procedure was performed for one-third of all samples in duplicate. The product of the restriction digest was verified on a $3 \%$ agarose gel (stained with Ethidium bromide) and underwent electrophoresis in a $1 \times$ TRIS-Borate-EDTA buffer at $120 \mathrm{~V}$ for $45 \mathrm{~min}$. Enzyme restriction generated six pieces. The difference between genotypes was determined by the fifth piece. The presence of a $\mathrm{G}$ at this piece generated a 327-bp fragment, whereas the 327-bp fragment was divided into 114- and 213-bp fragments when the fifth piece contained $\mathrm{T}$ heterozygous, TG genotype and three types of fragments - 327-, 213- and 114-bp fragments. The gels were visualised by UV light and the RFLP gel electrophoresis products were read by two independent people who were both unaware of the identities of samples. To test the AluI activity, a positive control was used for each run. In all, $20 \%$ of all samples were sequenced to confirm the accuracy of the genotyping process ${ }^{(21)}$.

\section{Dietary assessment}

All enrolled subjects received instructions to record their daily dietary intake nutrient for a 3-d 24-h dietary recall (2 weekdays and one during a weekend). Records were reviewed by a dietitian and analysed and were checked for clarification about food consumption and quantities. Analysis of the data was done to obtain fat intake percentage ${ }^{(22,23)}$.

\section{Statistical analysis}

First, for the evaluation of the normal distribution of quantitative variables, Shapiro-Wilk test was conducted. The total sample size with a two-sided $\alpha=0.05$ and $80 \%$ power $(\beta=0.2)$ could detect a Cohen's d effect size of 0.5 for differences in bone characteristics between groups with intake of fats. The differences between genotypes were assessed by one-way ANOVA test. The differences between groups with a high percentage of dietary fat intake (more than $30 \%$ of total energy content) and a low percentage of dietary fat intake $(\leq 30 \%$ of total energy content) were also assessed by independent-sample $t$ test and re-analysed by ANCOVA to adjust for the confounders effect, including age and dietary energy intake.

We considered BMD as a dependent variable and both visfatin genotype and fat intake as covariates in general linear model analysis to determine the interaction model. We included visfatin genotype, fat intake and visfatin genotype $\times$ fat intake in a custom model; also, we tabulated the CI by using estimate 


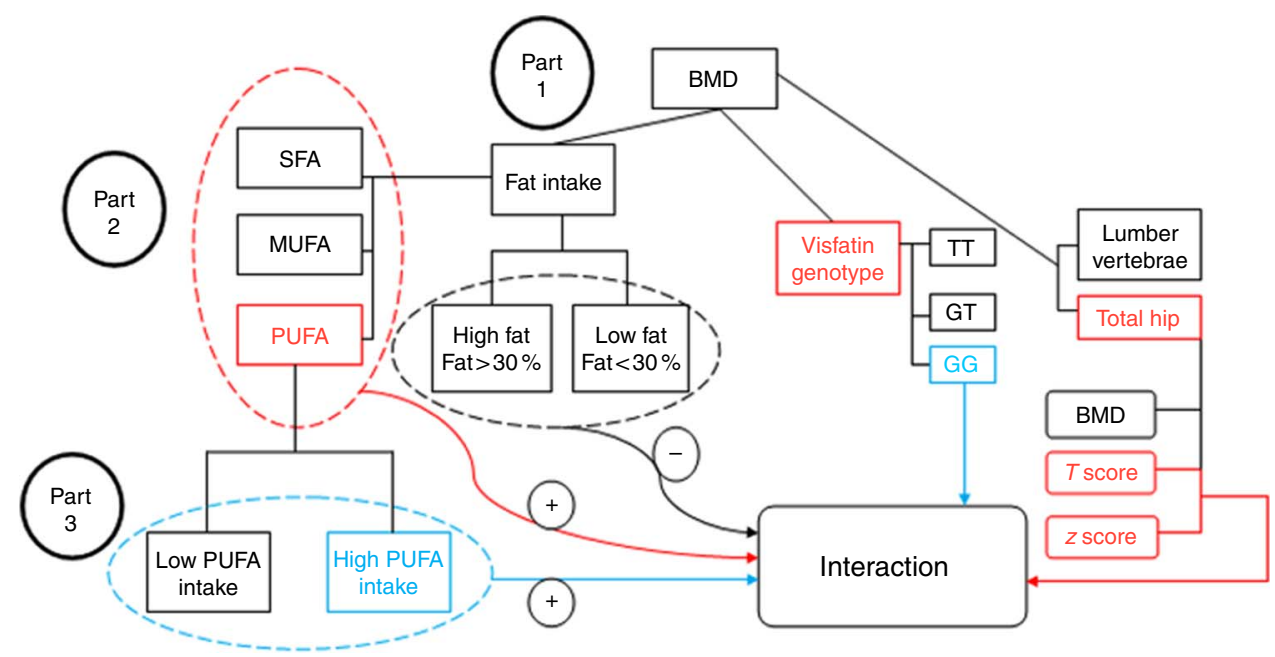

Fig. 1. Study diagram. First, the analysis investigated the participants' bone mineral density (BMD) status based on visfatin genotype and fat intake. Part 1: evaluation of the interaction between fat intake and BMD related to visfatin genotype; the results found no interaction between them (-) (by one-way ANOVA). Part 2: evaluation of the interaction based on fat type intake; an interaction was found between PUFA intake and hip $T$ score and $z$ score on the one hand, and visfatin genotype on the other $(+)$ (by general linear model (GLM) model). Part 3: determining the genotype determinative (GG) of this interaction ( +) based on the amount of PUFA intake (by GLM model).

parameter from option tab. Next, to find the significance, we evaluated the interaction based on type of fat intake such as median of PUFA, MUFA and SFA by the same direction.

The study diagram can be seen in Fig. 1 . The $t$ test was used to determine the contribution of each of the macronutrients between medians of total fat, PUFA, MUFA and SFA. The level of significance was set at a probability of $\leq 0.05$ for all tests. Statistical analysis was performed using SPSS version 22.0 (SPSS).

\section{Results}

\section{Clinical characteristics of the study participants}

The clinical and biochemical characteristics of 336 participants are shown in Table 1 . All the participants in the study were obese. Mean age, weight, height and BMI are 38.25 (SD 11.69) years, $82.88(\mathrm{SD} 14.06) \mathrm{kg}, 161.52(\mathrm{SD} 7.97) \mathrm{cm}$ and 31.79 (SD $4 \cdot 77) \mathrm{kg} / \mathrm{m}^{2}$, respectively (see Table 1 ).

\section{Characteristics of the study population based on visfatin genotypes}

All participants were categorised in three groups (GG, GT and TT) according to genotype. The characteristics of the participants, according to genotypes of the rs2110385 polymorphism, are shown in Table 2 . All analysed genotypes were distributed according to the Hardy-Weinberg equilibrium law in participants $(P>0.05)$. The frequencies of homozygous major allele (GG), heterozygous (GT) and homozygous minor allele of SNP ' $r$ 2 2110385 ' were 33.92, 48.51 and $17.54 \%$, respectively, with $86.6 \%$ of participants being women.

After genotype categorisation, significant differences were found between groups for age, height, weight, fat mass, TAG, HDL, T-cholesterol, fasting blood sugar (FBS) and LDL-cholesterol
$(P<0 \cdot 05)$. The results showed that lumbar BMD was significantly higher for participants with TT genotype $(P<0 \cdot 0001)$, similar to the lumbar $T$ score $(P<0 \cdot 0001)$. The present study shows significant $z$ score differences in both sites. We found higher $z$ score for subjects with the TT genotype at the hip site $(P=0 \cdot 019)$, and also at the lumbar region $(P<0 \cdot 0001)$. This study found significant differences between all types of dietary fat intake among genotypes of visfatin $(P<0.05)$. There were no significant differences for FFM, high sensitivity C-reactive protein (hs-CRP) and hip $T$ score between different genotype groups (Table 2 ).

\section{Characteristics of the study population, based on fat intake}

All 336 participants were divided into two groups based on fat intake ( $\leq 30 \%$ of total energy content $(23.7 \%$ of participants) and more than $30 \%$ of total energy intake $(76.3 \%$ of participants). Before adjustment for age and energy intake, significant differences between groups were found for age, weight, fat percentage, fat mass, visceral fat, TAG, HDL-cholesterol, lumbar BMD and $T$ score, as well as hip BMD and $T$ score $(P<0.05)$. After adjustment for age and energy intake, significantly higher levels of fat percentage, fat mass, visceral fat and hs-CRP $(P<0.05)$ were remained in women with high fat intake and the significant differences in BMD at both regions were lost (Table 3).

\section{Gene-diet interaction}

Using the general linear model, the interaction between visfatin genotypes and different types of dietary fat intake (PUFA, MUFA and SFA) on bone density was examined. The results showed there was an interaction between visfatin genotypes with dietary PUFA intake in terms of hip $T$ score and $z$ score ( $P=0.043, B=-0.08 ; P=0 \cdot 04, B=-0.078$, respectively) while we found any interaction between other types of dietary fat 
Table 1. Biochemical and clinical characteristics of the 336 study participants (Mean values and standard deviations)

\begin{tabular}{|c|c|c|c|c|}
\hline & Minimum & Maximum & Mean & SD \\
\hline \multicolumn{5}{|l|}{ Anthropometry } \\
\hline Age (years) & $19 \cdot 00$ & 69.00 & 38.25 & 11.69 \\
\hline Height $(\mathrm{cm})$ & 146.00 & 185.00 & $161 \cdot 52$ & $7 \cdot 97$ \\
\hline Weight (kg) & $61 \cdot 20$ & $127 \cdot 70$ & $82 \cdot 88$ & 14.06 \\
\hline BMI $\left(\mathrm{kg} / \mathrm{m}^{2}\right)$ & $25 \cdot 00$ & $46 \cdot 70$ & 31.79 & 4.77 \\
\hline Fat percentage & $15 \cdot 40$ & 51.00 & $37 \cdot 47$ & 7.44 \\
\hline FFM (kg) & 38.40 & 84.00 & 51.48 & $9 \cdot 28$ \\
\hline Fat mass $(\mathrm{kg})$ & 13.80 & 61.50 & 31.33 & 9.72 \\
\hline Visceral fat & 3.00 & 18.00 & 8.37 & 3.14 \\
\hline Trunk fat & 1.30 & 28.50 & 18.56 & 5.06 \\
\hline \multicolumn{5}{|l|}{ Dietary fat intake } \\
\hline Total fat $(\mathrm{g})$ & 60.55 & 133.83 & 82.74 & $17 \cdot 83$ \\
\hline SFA (g) & $10 \cdot 39$ & 38.78 & 21.66 & $6 \cdot 76$ \\
\hline MUFA (g) & 29.51 & 38.32 & 33.64 & 3.91 \\
\hline PUFA (g) & $13 \cdot 18$ & $50 \cdot 74$ & $25 \cdot 27$ & 9.83 \\
\hline \multicolumn{5}{|l|}{ Biochemical characteristics } \\
\hline TAG $(\mathrm{mmol} / \mathrm{l})$ & 38.00 & $347 \cdot 00$ & 128.07 & 52.97 \\
\hline Total cholesterol $(\mathrm{mmo} / \mathrm{l})$ & 99.00 & 270.00 & 182.06 & $34 \cdot 10$ \\
\hline HDL-cholesterol (mg/dl) & 24.00 & 97.00 & 46.00 & 11.69 \\
\hline LDL-cholesterol (mg/dl) & 44.00 & 176.00 & $102 \cdot 49$ & 24.52 \\
\hline hs-CRP (mg/l) & 0.10 & 17.50 & 3.12 & 3.63 \\
\hline Insulin (m IU/ml) & $2 \cdot 20$ & $42 \cdot 10$ & $12 \cdot 87$ & 6.57 \\
\hline FBS $(\mathrm{m} \mathrm{mol} / \mathrm{l})$ & 73.00 & 236.00 & $99 \cdot 47$ & $19 \cdot 16$ \\
\hline \multicolumn{5}{|l|}{ Bone densitometry } \\
\hline BMD L2-L4 g/cm² & 0.91 & 1.44 & 1.17 & 0.14 \\
\hline Z L2-L4 & $-2 \cdot 70$ & 1.20 & -0.68 & $1 \cdot 18$ \\
\hline T L2-L4 & -2.40 & $2 \cdot 10$ & -0.20 & $1 \cdot 23$ \\
\hline BMD Total $\mathrm{g} / \mathrm{cm}^{2}$ & 0.87 & 1.89 & $1 \cdot 10$ & 0.20 \\
\hline Z Total & -1.60 & $4 \cdot 20$ & 0.27 & $1 \cdot 15$ \\
\hline T Total & $-1 \cdot 20$ & $5 \cdot 10$ & 0.62 & 1.23 \\
\hline
\end{tabular}

FBS, fasting blood sugar; hs-CRP, high sensitivity C-reactive protein; FFM, fat-free mass; TBW, total body water; BMD, bone mineral density; total hip, L2-L4 (L) lumbar.

Table 2. Study population characteristics based on visfatin genotypes

(Mean values and standard deviations)

\begin{tabular}{|c|c|c|c|c|c|c|c|}
\hline & \multicolumn{2}{|c|}{$\mathrm{GG}(n 114)$} & \multicolumn{2}{|c|}{ GT $(n$ 163) } & \multicolumn{2}{|c|}{ TT $(n 59)$} & \multirow[b]{2}{*}{$P$} \\
\hline & Mean & SD & Mean & SD & Mean & SD & \\
\hline \multicolumn{8}{|l|}{ Anthropometry } \\
\hline Age (years) & 32.69 & $9 \cdot 81$ & 39.19 & 11.95 & 37.86 & 10.95 & $<0.0001^{*}$ \\
\hline Height $(\mathrm{cm})$ & $159 \cdot 15$ & 7.96 & 161.58 & $7 \cdot 71$ & $163 \cdot 20$ & 9.42 & $0.012^{*}$ \\
\hline Weight (kg) & 87.06 & $17 \cdot 25$ & $82 \cdot 67$ & $14 \cdot 20$ & $80 \cdot 42$ & 9.64 & $0.008^{*}$ \\
\hline FFM (kg) & 53.05 & 7.01 & 51.48 & 9.43 & $50 \cdot 12$ & $10 \cdot 42$ & 0.102 \\
\hline Fat mass $(\mathrm{kg})$ & 34.03 & 13.79 & 31.11 & $9 \cdot 12$ & $30 \cdot 20$ & 9.14 & $0.038^{*}$ \\
\hline \multicolumn{8}{|l|}{ Dietary fat intake } \\
\hline Total fat $(\mathrm{g})$ & $78 \cdot 20$ & 7.62 & 84.11 & 20.02 & $80 \cdot 19$ & 13.49 & $0.0037^{\star}$ \\
\hline SFA (g) & 28.05 & $5 \cdot 60$ & $19 \cdot 79$ & 5.63 & 24.87 & 9.63 & $<0.0001^{*}$ \\
\hline MUFA $(\mathrm{g})$ & 30.12 & $5 \cdot 27$ & 34.46 & 9.53 & 33.08 & 17.83 & $0.0032^{*}$ \\
\hline PUFA $(\mathrm{g})$ & 20.01 & 3.91 & $26 \cdot 70$ & 1.01 & $23 \cdot 30$ & $1 \cdot 25$ & $<0.0001^{*}$ \\
\hline \multicolumn{8}{|l|}{ Biochemical characteristics } \\
\hline TAG $(\mathrm{mmol} / \mathrm{l})$ & $109 \cdot 25$ & $40 \cdot 14$ & 135.69 & 55.59 & $102 \cdot 00$ & 33.94 & $<0.0001^{*}$ \\
\hline Total cholesterol $(\mathrm{mmo} / \mathrm{l})$ & $170 \cdot 42$ & $40 \cdot 00$ & $184 \cdot 70$ & 33.14 & $177 \cdot 07$ & $34 \cdot 22$ & $<0.0001^{*}$ \\
\hline HDL-cholesterol (mg/dl) & $45 \cdot 75$ & 8.94 & $44 \cdot 88$ & $10 \cdot 97$ & $52 \cdot 26$ & $15 \cdot 63$ & $<0.0001^{*}$ \\
\hline LDL-cholesterol (mg/dl) & 93.33 & 27.84 & 104.94 & 24.06 & 96.60 & $23 \cdot 10$ & $0.0006^{*}$ \\
\hline hs-CRP (mg/l) & 2.95 & 3.32 & 3.13 & 3.56 & $3 \cdot 20$ & 4.43 & 0.887 \\
\hline Insulin (m IU/ml) & $10 \cdot 73$ & 4.56 & $13 \cdot 27$ & 6.98 & $12 \cdot 48$ & 5.47 & $0.002^{*}$ \\
\hline FBS $(\mathrm{mmol} / \mathrm{l})$ & 92.00 & 9.72 & 101.09 & $20 \cdot 73$ & 96.73 & $14 \cdot 38$ & $<0.0001^{*}$ \\
\hline \multicolumn{8}{|l|}{ Bone densitometry } \\
\hline BMD L2-L4 & 1.05 & 0.09 & $1 \cdot 19$ & 0.14 & 1.22 & 0.14 & $<0.0001^{*}$ \\
\hline$z$ score L2-L4 & -2.02 & 0.46 & -0.59 & $1 \cdot 14$ & 0.15 & 0.90 & $<0.0001^{\star}$ \\
\hline$T$ score L2-L4 & $-1 \cdot 20$ & 0.78 & -0.07 & 1.26 & 0.15 & $1 \cdot 19$ & $<0.0001^{*}$ \\
\hline BMD Total & 1.06 & 0.13 & $1 \cdot 11$ & 0.23 & 1.05 & 0.04 & $0.022^{*}$ \\
\hline z score Total & -0.02 & 0.86 & 0.31 & 1.26 & 0.37 & 0.87 & $0.019^{*}$ \\
\hline$T$ score Total & 0.55 & $1 \cdot 10$ & 0.67 & $1 \cdot 38$ & 0.42 & 0.38 & 0.343 \\
\hline
\end{tabular}

FBS, fasting blood sugar; hs-CRP, high sensitivity C-reactive protein; FFM, fat-free mass; TBW, total body water; PTH, parathyroid hormone; BMD, bone mineral density; Total hip, L2-L4(L) lumbar. 
Table 3. Characteristics based on dietary fat intake: $\leq 30 \%$ of total energy intake $v .>30 \%$ of total energy intake (Mean values and standard deviations)

\begin{tabular}{|c|c|c|c|c|c|c|}
\hline & \multicolumn{2}{|c|}{ Low fat intake } & \multicolumn{2}{|c|}{ High fat intake } & \multirow[b]{2}{*}{$P$} & \multirow[b]{2}{*}{$P+$} \\
\hline & Mean & SD & Mean & SD & & \\
\hline \multicolumn{7}{|l|}{ Anthropometry } \\
\hline Age (years) & 46.58 & $10 \cdot 05$ & 38.58 & 11.64 & $<0.0001$ & * \\
\hline Height (cm) & $161 \cdot 00$ & $7 \cdot 61$ & $161 \cdot 30$ & 67.69 & 0.96 & \\
\hline Weight (kg) & $85 \cdot 60$ & 11.69 & 94.77 & $15 \cdot 48$ & $<0.0001$ & 0.31 \\
\hline Fat percentage & $36 \cdot 74$ & $7 \cdot 72$ & 41.00 & $6 \cdot 18$ & $<0.0001$ & $0.009^{*}$ \\
\hline FFM (kg) & $54 \cdot 32$ & 11.48 & 55.95 & 11.56 & 0.21 & 0.55 \\
\hline Fat mass (kg) & $31 \cdot 28$ & 6.93 & 38.82 & 8.63 & $<0.0001$ & $0.01^{*}$ \\
\hline Visceral fat & 9.75 & 3.01 & $11 \cdot 16$ & $3 \cdot 61$ & 0.0004 & $0.008^{*}$ \\
\hline \multicolumn{7}{|l|}{ Biochemistry characteristic } \\
\hline TAG $(\mathrm{mmol} / \mathrm{l})$ & $123 \cdot 75$ & 43.67 & $139 \cdot 60$ & $69 \cdot 21$ & 0.02 & 0.47 \\
\hline Total cholesterol (mmo/l) & $199 \cdot 87$ & $30 \cdot 24$ & $192 \cdot 44$ & $36 \cdot 25$ & 0.06 & 0.95 \\
\hline HDL-cholesterol (mmo/l) & $57 \cdot 50$ & $19 \cdot 77$ & 51.02 & 12.99 & 0.0001 & $0 \cdot 76$ \\
\hline LDL-cholesterol (mmo/l) & $107 \cdot 75$ & $15 \cdot 98$ & $103 \cdot 46$ & 23.69 & 0.08 & 0.74 \\
\hline hs-CRP (mg/l) & 1.33 & 0.71 & $4 \cdot 28$ & 3.96 & $<0.0001$ & $0.05^{\star}$ \\
\hline Insulin (mlU/ml) & $11 \cdot 26$ & $4 \cdot 33$ & 15.54 & 6.96 & $<0.0001$ & 0.17 \\
\hline FBS $(\mathrm{mmol} / \mathrm{l})$ & 89.75 & 11.63 & $112 \cdot 31$ & $51 \cdot 33$ & $<0.0001$ & $0 \cdot 28$ \\
\hline \multicolumn{7}{|l|}{ Bone densitometry } \\
\hline L2-L4 BMD & $1 \cdot 16$ & 0.13 & $1 \cdot 21$ & $0 \cdot 16$ & 0.004 & 0.93 \\
\hline L2-L4 z score & -0.56 & $1 \cdot 21$ & -0.64 & $1 \cdot 25$ & 0.56 & 0.67 \\
\hline L2-L4 T score & -0.32 & 1.06 & 0.08 & $1 \cdot 30$ & 0.004 & 0.92 \\
\hline Total BMD & 1.06 & 0.14 & $1 \cdot 10$ & $0 \cdot 16$ & 0.02 & 0.51 \\
\hline Total $z$ score & 0.22 & 0.87 & 0.13 & 0.96 & 0.39 & 0.71 \\
\hline Total $T$ score & 0.34 & 0.88 & 0.58 & 1.02 & 0.03 & 0.45 \\
\hline
\end{tabular}

FBS, fasting blood sugar; hs-CRP, high sensitivity C-reactive protein; FFM, fat-free mass; TBW, total body water; PTH, parathyroid hormone; BMD, bone mineral density; Total hip, L2-L4(L) lumbar.

${ }^{*} P<0.05$.

$\dagger$ After adjustment for age and total energy intake.

Table 4. Evaluation of interaction between type of fat intake and visfatin genotypes on bone mineral density ( $\beta$-Coefficients and $95 \%$ confidence intervals)

\begin{tabular}{|c|c|c|c|c|c|c|c|}
\hline & Sig. & $\beta$ & $95 \% \mathrm{Cl}$ & & Sig & $\beta$ & $95 \% \mathrm{Cl}$ \\
\hline Total fat $\times$ visfatin genotypes & & & & PUFA $\times$ visfatin genotypes & & & \\
\hline BMD Total & 0.34 & -0.007 & $-0.02,0.008$ & BMD Total & 0.17 & -0.012 & $-0.30,0.006$ \\
\hline$T$ score Total & 0.10 & -0.05 & $-0.11,0.01$ & $T$ score Total & $0.043^{\star}$ & -0.08 & $-0.15,0.003$ \\
\hline$z$ score Total & 0.53 & -0.05 & $-0.11,0.001$ & $z$ score Total & $0.04^{\star}$ & -0.078 & $-0.15,0.004$ \\
\hline BMD L2-L4 & 0.58 & 0.003 & $-0.008,0.014$ & BMD L2-L4 & 0.96 & $<0.0001$ & $-0.013,0.014$ \\
\hline$T$ score L2-L4 & 0.59 & -0.023 & $-0.067,0.11$ & $T$ score L2-L4 & 0.97 & 0.002 & $-0.11,0.11$ \\
\hline z score L2-L4 & 0.57 & 0.02 & $-0.09,0.10$ & z score L2-L4 & 0.99 & $<0.0001$ & $-0.10,0.10$ \\
\hline SFA $\times$ visfatin genotypes & & & & MUFA $\times$ visfatin genotypes & & & \\
\hline BMD Total & 0.78 & 0.003 & $-0.020,0.026$ & BMD Total & 0.98 & $<0.0001$ & $-1 \cdot 12,1 \cdot 13$ \\
\hline$T$ score Total & 0.58 & 0.02 & $-0.07,0.13$ & $T$ score Total & 0.74 & 0.026 & $-0.13,0.18$ \\
\hline$z$ score Total & 0.96 & 0.002 & $-0.09,0.10$ & $z$ score Total & 0.99 & $<0.0001$ & $-0.15,0.15$ \\
\hline BMD L2-L4 & 0.088 & 0.013 & $-0.002,0.029$ & BMD L2-L4 & 0.81 & 0.003 & $-0.29,0.23$ \\
\hline$T$ score L2-L4 & 0.084 & 0.11 & $-0.017,0.24$ & T score L2-L4 & 0.80 & 0.09 & $-0.21,0.03$ \\
\hline z score L2-L4 & 0.14 & 0.087 & $-0.03,0.20$ & z score L2-L4 & 0.48 & -0.068 & $-0.26,0.12$ \\
\hline
\end{tabular}

BMD, bone mineral density; Total hip, L2-L4 (L) lumbar; visfatin genotype; GG, GT, TT. Custom model in general linear model; bone mineral density as a dependent model, visfatin Genotype $\times$ Medians intake PUFA. Data were extracted from parameter estimates by general linear model.

${ }^{*} P<0.05$.

intake such as MUFA, SFA also total dietary fat intake with visfatin genotype on BMD, $T$ score and $z$ score of hip and lumbar (Table 4). Following the classification of participants into two groups based on median PUFA intake, we found that participants with GG genotype who had high PUFA diets had lower hip $T$ scores and $z$ scores; however, both other genotypes showed changes in different directions (Fig. 2). On the other hand, to clarify the effect of other dietary intakes, we used independent-samples $t$ tests to evaluate the difference of macronutrients and kcal $(\mathrm{kJ})$ intakes among both high and low medians of different types of dietary fat intake. As shown in
Table 5, participants who were in the high PUFA intake group had lower energy $(P=0.02)$ and protein $(P=0.0016)$ intake than those in the low PUFA intake group, but there was a significant difference between carbohydrate intake level among medians of PUFA intake (Table 5).

\section{Discussion}

According to the results, it was found that women with TT genotype had higher lumbar BMD, whereas those with GT genotype had higher hip BMD. In examining the interaction 

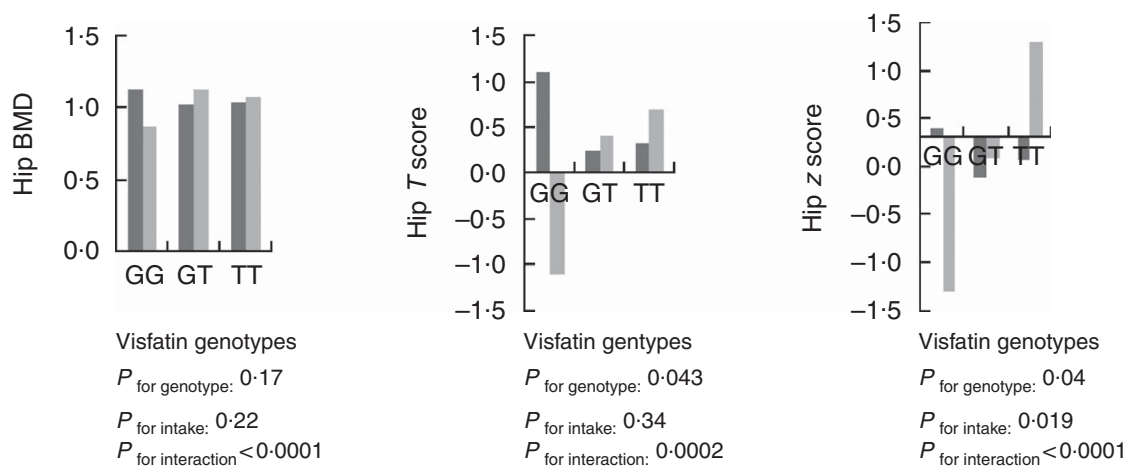

Fig. 2. Interaction between amount of PUFA intake and visfatin genotype ( $r \mathrm{~s} 2110385)$ on hip bone mineral density $\left(\mathrm{g} / \mathrm{cm}^{2}\right), T$ score and $z$ score. Custom model in general linear model; BMD is considered as a dependent variable in this model, and PUFA, visfatin genotype and genotype $\times$ intake PUFA are covariate. $\square$, Low PUFA intake; $\square$, high PUFA intake. * $P<0.05$.

Table 5. Distribution of macronutrients based on types of dietary fat intake†‡ (Mean values and standard deviations)

\begin{tabular}{|c|c|c|c|c|c|c|c|c|c|c|}
\hline & \multicolumn{4}{|c|}{ Total fat } & \multirow[b]{3}{*}{$P$} & \multicolumn{4}{|c|}{ PUFA } & \multirow[b]{3}{*}{$P$} \\
\hline & \multicolumn{2}{|c|}{ Low } & \multicolumn{2}{|c|}{ High } & & \multicolumn{2}{|c|}{ Low } & \multicolumn{2}{|c|}{ High } & \\
\hline & Mean & SD & Mean & SD & & Mean & SD & Mean & SD & \\
\hline kJ & $8568 \cdot 8$ & 2083.6 & 8171.0 & 2585.7 & $0.0028^{*}$ & $8581 \cdot 4$ & $2163 \cdot 1$ & $8159 \cdot 0$ & $2518 \cdot 8$ & $0.02^{\star}$ \\
\hline kcal & $2048 \cdot 7$ & $498 \cdot 4$ & 1953 & $618 \cdot 1$ & $0.0028^{*}$ & 2051.9 & $517 \cdot 1$ & $1950 \cdot 0$ & $602 \cdot 3$ & $0.02^{*}$ \\
\hline Cho & 289.2 & 30.5 & 233.5 & 30.1 & $<0.0001^{*}$ & $278 \cdot 1$ & 37.8 & $244 \cdot 2$ & 37.5 & 0.45 \\
\hline $\mathrm{Pr}$ & $62 \cdot 3$ & $17 \cdot 8$ & $61 \cdot 2$ & 14.9 & $0.01^{*}$ & 64.2 & $18 \cdot 0$ & 59.4 & $14 \cdot 3$ & $0.0016^{\star}$ \\
\hline \multirow[t]{2}{*}{ Fat } & 67.9 & 8.5 & 93.4 & $11 \cdot 7$ & $<0.0001^{*}$ & 71.9 & $12 \cdot 7$ & 89.4 & $15 \cdot 0$ & $0.01^{*}$ \\
\hline & \multicolumn{4}{|c|}{ MUFA } & & \multicolumn{4}{|c|}{ SFA } & \\
\hline kJ & 8543.7 & $2326 \cdot 3$ & $8196 \cdot 4$ & 2376.5 & 0.39 & 8518.6 & $2008 \cdot 3$ & 8222 & $2648 \cdot 4$ & $0.0002^{*}$ \\
\hline kcal & $2042 \cdot 4$ & $556 \cdot 9$ & $1959 \cdot 2$ & $568 \cdot 2$ & 0.39 & 2036.5 & $480 \cdot 8$ & 1965 & 633.1 & $0.0002^{*}$ \\
\hline Cho & $281 \cdot 8$ & 39.2 & $240 \cdot 6$ & $32 \cdot 0$ & $<0.0001^{*}$ & $275 \cdot 8$ & 34.3 & 246.5 & $42 \cdot 4$ & $<0.0001^{*}$ \\
\hline $\mathrm{Pr}$ & $64 \cdot 1$ & $20 \cdot 1$ & 59.1 & $11 \cdot 2$ & $<0.0001^{*}$ & $60 \cdot 4$ & $1 \cdot 7$ & 63.0 & $15 \cdot 2$ & $<0.0001^{*}$ \\
\hline Fat & $70 \cdot 0$ & $11 \cdot 3$ & $91 \cdot 3$ & $13 \cdot 5$ & $<0.0001^{*}$ & 74.9 & $12 \cdot 6$ & $86 \cdot 5$ & $17 \cdot 7$ & $<0.0001^{*}$ \\
\hline
\end{tabular}

Cho, carbohydrate; Pr, protein.

${ }^{*} P<0.05$.

$\dagger t$ Test analysis, all variables adjusted by energy.

$\ddagger$ Medians of PUFA $(22.8 \mathrm{~g})$, MUFA (33 g), SFA $(19.7 \mathrm{~g})$ and total fat $(79.1 \mathrm{~g})$.

between fat intake and BMD among genotypes of visfatin, the percentage and type of dietary fat intake was investigated. High and low percentages of fat intake did not play a crucial role, but study results were different for various types of fat. This means that women with GG genotype who consumed high-PUFA diets had lower bone density at total hip. However, for the other two genotypes, PUFA intake and bone density were directly related at the hip site, but this interaction was not found at the lumbar spine. This study was the second one to investigate the association between visfatin genotype and BMD. In a similar study, Mirzaei et al. ${ }^{(21)}$ examined diabetic patients with regard to the relationship between visfatin genotypes and BMD; it was found that diabetic patients with GG genotype had significantly lower hip $z$ scores.

Another finding of this study indicated that women with GT genotype had significantly higher TAG, total cholesterol, FBS, insulin and lower HDL, compared with both other genotypes. A recent study ${ }^{(21)}$ found a significant difference in HDL similar to this study, but no other association with other lipid profile components was reported. In contrast to our research, Rehiem \& Gado ${ }^{(24)}$ carried out a case-control study among an Egyptian population, and found that individuals with the GG genotype had higher HDL.

By first categorising participants based on percentage of fat intake, it was indicated that - after adjustment for age and total energy, through a linear regression model - there were no significant differences between BMD and fat intake at three regions based on visfatin genotypes. However, significant relationships were found between hs-CRP, $25(\mathrm{OH})_{2} \mathrm{D}$, fat percentage, fat mass, visceral fat and fat intake. Somewhat similar to the present study, previous experiments have indicated a positive association between high fat intake and fat mass, fat percentage and visceral adiposity ${ }^{(25-27)}$. A large number of studies found that high-fat diets increased hs-CRP ${ }^{(28)}$, and also the absorption and bioavailability of vitamin $\mathrm{D}$, which was similar to our results ${ }^{(29-32)}$.

In the second part, fat type interaction related to BMD and visfatin genotype was examined. The result was that an inverse 
association between high-PUFA diets and hip $z$ score was found among women with GG genotype; this proved to be different from both other genotypes. Previous studies have shown paradoxical results related to the effect of PUFA intake on bone density. Harris et $a l^{(33)}$, for example, found a negative association between dietary PUFA intake and BMD at the spine, total body and Ward's triangle BMD, but only among postmenopausal women who were using hormone therapy (HT). However, in a group without HT, no significant association was seen. In another study carried out by Järvinen et $a l^{(34)}$, a significant positive effect of PUFA on lumbar bone status in women without hormone therapy was demonstrated. Recent studies have also indicated that various types of PUFA intake, such as linolenic $\operatorname{acid}^{(35,36)}$ and arachidonic $\operatorname{acid}^{(37)}$, were associated with reduced hip fracture rates. Similarly, Virtanen et $a l .{ }^{(38)}$ found no significant relationship between PUFA and hip fracture rates among either sex in a cohort study, but low PUFA intake (especially linoleic acid) was considered as a risk factor of hip fracture only for women ${ }^{(39)}$. To evaluate the effect of dietary intake such as carbohydrate, protein and fat, a oneway ANOVA was used. Previous studies have shown positive effects of high protein intake and increased $\mathrm{BMD}^{(40,41)}$. The present study found participants who were in the high PUFA intake group had low intake of energy and protein, and so we concluded that protein and energy intakes could not explain the high PUFA intakes.

As mentioned above, the significant effect of PUFA intake on $\mathrm{BMD}$ is not clear, and the evidence is controversial. In order to further examine this, visfatin was considered as an efficient adipokine related to dietary fat metabolism, and thus evaluating visfatin genotypes seemed promising. Previous studies had suggested an effective role of visfatin serum level on bone status among patients with inflammatory bowel disease ${ }^{(42)}$ and cancer patients $^{(43)}$, but in opposition to these recent publications, Iacobellis et $a l^{(13)}$ and Sucunza et $a l^{(12)}$ - who investigated patients with the metabolic syndrome and acromegaly, respectively - both found a negative association of visfatin with BMD. On the other hand, Tohidi et $a l^{(44)}$ and Zhang et $a l^{(45)}$ who worked on postmenopausal women, and Peng et al. ${ }^{(46)}$, whose focus was on men, found no correlation between BMD and visfatin. Given that, it has been shown that visfatin has many effects on fat and glucose metabolic pathways ${ }^{(15)}$, insulin resistance ${ }^{(47)}$ and lipid profiles in adult patients with diabetes ${ }^{(21)}$, obesity ${ }^{(48)}$ and inflammation ${ }^{(49)}$. It seems that owing to inconsistencies between studies, it may be necessary to examine the genetic diversity among the population. Following our previous study ${ }^{(50)}$ on the relationship between visfatin genotype and BMD among diabetic patients, the present study was carried out with the aim of examining dietary fat intake in relation to the correlation between BMD and visfatin genotype. Women with GG genotype who had high PUFA intake diets had lower hip $z$ scores and $T$ scores.

The current study has a number of limitations. All participants in the study were overweight or obese; therefore, the findings from the present study may not be generalisable to individuals with low or normal BMI. There were no longitudinal data in order to determine any longer-term effects of PUFA on BMD among different genotypes. The daily routine intakes of all participants were monitored, which perhaps changed the results, compared with a case-control or clinical study. Any changes about PUFA intake and BMD over time could not be reflected in this crosssectional study because they were assessed only with single measurements. As a nutrigenomics study, no previous study has investigated the influence of visfatin genotype related to effect of types of dietary fat intake on BMD. Previous studies had researched the effect of PUFA on BMD, and as the results were inconsistent the present study is the first one that examined visfatin genotypes as an interaction study.

It is concluded that subjects with GG genotype and high PUFA diets had lower hip $z$ scores and $T$ scores, unlike the other genotypes. Understanding the complex mechanisms of these changes needs more randomised, controlled future studies among larger populations to examine the interaction of molecular and metabolic mechanisms.

\section{Acknowledgements}

The authors acknowledge Tehran University of Medical Sciences and Osteoporosis Research Center, Tehran, Iran, for allowing us to conduct this cross-sectional study.

This work was supported by the Tehran University of Medical Sciences (grant no.: 94-02-161-29564)

Study concept and design and study supervisions: K. M.; acquisition of data, critical revision of the manuscript for important intellectual content and administrative, technical and material support: K. M. and L. K.; analysis and interpretation of data: K. M. and S. A. K.; drafting of the manuscript: L. K. and Z. M.; statistical analysis: K. M. and Z. M.

The authors declare that there are no conflicts of interest.

\section{References}

1. Patel R, Rodriguez A, Yasmeen T, et al. (2015) Impact of obesity on osteoporosis: limitations of the current modalities of assessing osteoporosis in obese subjects. Clin Rev Bone Miner Metab 13, 36-42.

2. Migliaccio S, Greco EA, Fornari R, et al. (2011) Is obesity in women protective against osteoporosis. Diabetes Metab Syndr Obes 4, 273-282.

3. Zhao LJ, Jiang H, Papasian CJ, et al. (2008) Correlation of obesity and osteoporosis: effect of fat mass on the determination of osteoporosis. J Bone Miner Res 23, 17-29.

4. Yerges-Armstrong LM, Miljkovic I, Cauley JA, et al. (2010) Adipose tissue and volumetric bone mineral density of older Afro-Caribbean men. J Bone Miner Res 25, 2221-2228.

5. Wang L, Wang W, Xu L, et al. (2013) Relation of visceral and subcutaneous adipose tissue to bone mineral density in Chinese women. Int J Endocrinol 2013, 378632.

6. Salamat MR, Salamat AH, Abedi I, et al. (2013) Relationship between weight, body mass index, and bone mineral density in men referred for dual-energy X-ray absorptiometry scan in Isfahan, Iran. J Osteoporos 2013, 205963.

7. Kim SJ, Yang W-G, Cho E, et al. (2012) Relationship between weight, body mass index and bone mineral density of lumbar spine in women. $J$ Bone Metab 19, 95-102.

8. Cobayashi F, Lopes LA \& Taddei JA (2005) Bone mineral density in overweight and obese adolescents. J Pediatr (Rio J) 81, 337-342.

9. Castro JP, Joseph LA, Shin JJ, et al. (2005) Differential effect of obesity on bone mineral density in White, Hispanic and 
African American women: a cross sectional study. Nutr Metab (Lond) 2, 9.

10. Siviero-Miachon AA, Spinola-Castro AM, de Martino Lee ML, et al. (2015) Visfatin is a positive predictor of bone mineral density in young survivors of acute lymphocytic leukemia. J Bone Miner Metab 35, 73-82.

11. Maeda K, Okubo K, Shimomura I, et al. (1996) cDNA cloning and expression of a novel adipose specific collagenlike factor, apM1 (AdiPoseMost abundant Gene transcript 1). Biochem Biophys Res Commun 221, 286-289.

12. Sucunza N, Barahona MJ, Resmini E, et al. (2009) A link between bone mineral density and serum adiponectin and visfatin levels in acromegaly. J Clin Endocrinol Metab 94, 3889-3896.

13. Iacobellis G, Iorio M, Napoli N, et al. (2011) Relation of adiponectin, visfatin and bone mineral density in patients with metabolic syndrome. J Endocrinol Invest 34, e12-e15.

14. Hossein-Nezhad A, Hosseinzadeh-Attar M, Najmafshar A, et al. (2009) Relationship between genotype and serum levels of adipokines and bone mineral density in type 2 diabetes mellitus patients. Iran J Diabetes Metab Disorders $\mathbf{8}$, 77-86.

15. Jian WX, Luo TH, Gu YY, et al. (2006) The visfatin gene is associated with glucose and lipid metabolism in a Chinese population. Diabet Med 23, 967-973.

16. Basaran S, Guzel R, Coskun-Benlidayi I, et al. (2007) Vitamin D status: effects on quality of life in osteoporosis among Turkish women. Qual Life Res 16, 1491-1499.

17. Park S, Kim M, Baik S, et al. (2013) Gestational diabetes is associated with high energy and saturated fat intakes and with low plasma visfatin and adiponectin levels independent of prepregnancy BMI. Eur J Clin Nutr 67, 196-201.

18. Haghighatdoost F, Hosseinzadeh-Attar MJ, Kabiri A, et al. (2012) Effect of substituting saturated with monounsaturated fatty acids on serum visfatin levels and insulin resistance in overweight women: a randomized cross-over clinical trial. Int J Food Sci Nutr 63, 772-781.

19. de Luis DA, Aller R, Gonzalez Sagrado M, et al. (2010) Serum visfatin concentrations are related to dietary intake in obese patients. Ann Nutr Metab 57, 265-270.

20. Körner A, Böttcher Y, Enigk B, et al. (2007) Effects of genetic variation in the visfatin gene (PBEF1) on obesity, glucose metabolism, and blood pressure in children. Metabolism 56, 772-777.

21. Mirzaei K, Hossein-Nezhad A, Javad H-AM, et al. (2009) Visfatin genotype may modify the insulin resistance and lipid profile in type 2 diabetes patients. Minerva Endocrinol 34, 273-279.

22. Pereira RA, Araujo MC, Lopes TdS, et al. (2010) How many 24-hour recalls or food records are required to estimate usual energy and nutrient intake? Cad Saude Publica 26, 2101-2111.

23. Shim J-S, Oh K \& Kim HC (2014) Dietary assessment methods in epidemiologic studies. Epidemiol Health 36, e2014009.

24. Rehiem HA \& Gado T (2010) Visfatin G-948T polymorphism in Egyptian type 2 diabetes. $J$ Am Sci 6, 77-86.

25. Neville MM, Geppert J, Min Y, et al. (2012) Dietary fat intake, body composition and blood lipids of university men and women. Nutr Health 21, 173-185.

26. Kratz M, Baars $\mathrm{T}$ \& Guyenet S (2013) The relationship between high-fat dairy consumption and obesity, cardiovascular, and metabolic disease. Eur J Nutr 52, 1-24.

27. Bowen L, Taylor AE, Sullivan R, et al. (2015) Associations between diet, physical activity and body fat distribution: a cross sectional study in an Indian population. BMC Public Health 15, 281.
28. Rankin JW \& Turpyn AD (2007) Low carbohydrate, high fat diet increases C-reactive protein during weight loss. J Am Coll Nutr 26, 163-169.

29. Raimundo FV, Faulhaber GAM, Menegatti PK, et al. (2011) Effect of high-versus low-fat meal on serum 25-hydroxyvitamin D levels after a single oral dose of vitamin D: a single-blind, parallel, randomized trial. Int $J$ Endocrinol 2011, e2014009.

30. Dawson-Hughes B, Harris SS, Lichtenstein AH, et al. (2015) Dietary fat increases vitamin D-3 absorption. J Acad Nutr Diet 115, 225-230.

31. Iqbal J \& Hussain MM (2009) Intestinal lipid absorption. Am J Physiol Endocrinol Metab 296, E1183-E1194.

32. McLarnon A (2011) Nutrition: dietary fat might influence serum vitamin D level. Nat Rev Endocrinol 7, 562

33. Harris M, Farrell V, Houtkooper L, et al. (2015) Associations of polyunsaturated fatty acid intake with bone mineral density in postmenopausal women. J Osteoporos 2015, 809069.

34. Järvinen R, Tuppurainen M, Erkkilä A, et al. (2012) Associations of dietary polyunsaturated fatty acids with bone mineral density in elderly women. Eur J Clin Nutr 66, 496-503.

35. Orchard TS, Steven W, Lu B, et al. (2013) The association of red blood cell $n-3$ and $n-6$ fatty acids with bone mineral density and hip fracture risk in the women's health initiative. J Bone Miner Res 28, 505-515.

36. Farina EK, Kiel DP, Roubenoff R, et al. (2011) Dietary intakes of arachidonic acid and $\alpha$-linolenic acid are associated with reduced risk of hip fracture in older adults. J Nutr $\mathbf{1 4 1}$, $1146-1153$

37. Farina EK, Kiel DP, Roubenoff R, et al. (2012) Plasma phosphatidylcholine concentrations of polyunsaturated fatty acids are differentially associated with hip bone mineral density and hip fracture in older adults: the Framingham Osteoporosis Study. J Bone Miner Res 27, 1222-1230.

38. Virtanen JK, Mozaffarian D, Willett WC, et al. (2012) Dietary intake of polyunsaturated fatty acids and risk of hip fracture in men and women. Osteoporos Int 23, 2615-2624.

39. Virtanen JK, Mozaffarian D, Cauley JA, et al. (2010) Fish consumption, bone mineral density, and risk of hip fracture among older adults: the cardiovascular health study. J Bone Miner Res 25, 1972-1979.

40. Rapuri PB, Gallagher JC \& Haynatzka V (2003) Protein intake: effects on bone mineral density and the rate of bone loss in elderly women. Am J Clin Nutr 77, 1517-1525.

41. Promislow JH, Goodman-Gruen D, Slymen DJ, et al. (2002) Protein consumption and bone mineral density in the elderly: the Rancho Bernardo Study. Am J Epidemiol 155, 636-644.

42. Terzoudis S, Malliaraki N, Damilakis J, et al. (2016) Chemerin, visfatin, and vaspin serum levels in relation to bone mineral density in patients with inflammatory bowel disease. Eur J Gastroenterol Hepatol 28, 814-819.

43. Siviero-Miachon AA, Spinola-Castro AM, de Martino Lee ML, et al. (2015) Visfatin is a positive predictor of bone mineral density in young survivors of acute lymphocytic leukemia. J Bone Miner Metab 140, 1839-1845.

44. Tohidi M, Akbarzadeh S, Larijani B, et al. (2012) Omentin-1, visfatin and adiponectin levels in relation to bone mineral density in Iranian postmenopausal women. Bone $\mathbf{5 1}$, 876-881.

45. Zhang H, Xie H, Zhao Q, et al. (2010) Relationships between serum adiponectin, apelin, leptin, resistin, visfatin levels and bone mineral density, and bone biochemical markers in post-menopausal Chinese women. J Endocrinol Invest 33, 707-711.

46. Peng X-D, Xie H, Zhao Q, et al. (2008) Relationships between serum adiponectin, leptin, resistin, visfatin levels and bone 
mineral density, and bone biochemical markers in Chinese men. Clin Chim Acta 387, 31-35.

47. Zhang YY, Gottardo L, Thompson R, et al. (2006) A visfatin promoter polymorphism is associated with low-grade inflammation and type 2 diabetes. Obesity 14, 2119-2126.

48. Blakemore AI, Meyre D, Delplanque J, et al. (2009) A rare variant in the visfatin gene (NAMPT/PBEF1) is associated with protection from obesity. Obesity 17, 1549-1553.
49. Tabassum R, Mahendran Y, Dwivedi OP, et al. (2012) Common variants of IL6, LEPR, and PBEF1 are associated with obesity in Indian children. Diabetes 61, 626-631.

50. Hossein-Nezhad A, Hosseinzadeh-Attar M, Najmafshar A, et al. (2009) Relationship between genotype and serum levels of adipokines and bone mineral density in type 2 diabetes mellitus patients. J Diabetes Metab Disord $\mathbf{8}, 10$ 